\title{
Erratum to: Chemical Screening for Flagella-Associated Phenotypes in Chlamydomonas reinhardtii
}

\author{
Brittany Jack and Prachee Avasthi
}

\section{Erratum to:}

Chapter 17 in: Friedrich Fauser and Martin Jonikas (eds.), Plant Chemical Genomics: Methods and Protocols, Methods in Molecular Biology, vol. 1795, https://doi.org/10.1007/978-1-4939-7874-8_17

The original version of this chapter was inadvertently published with the incorrect affiliation for Brittany Jack and Dr. Prachee Avasthi. This has been corrected and the affiliation details has been updated as follows:

Brittany Jack - "Anatomy and Cell Biology Department, The University of Kansas Medical Center, Kansas City, KS, USA"

Prachee Avasthi - "Anatomy and Cell Biology Department, The University of Kansas Medical Center, Kansas City, KS, USA; Department of Ophthalmology, The University of Kansas Medical Center, Kansas City, KS, USA"

The updated online version of this chapter can be found at https://doi.org/10.1007/978-1-4939-7874-8_17

The updated online version of this book can be found at https://doi.org/10.1007/978-1-4939-7874-8 